\title{
Fundamental Study for New Evaluation Method Based on Physical and Psychological Load in Human Movement
}

\author{
Hiroaki Inoue and Shunji Shimizu \\ Tokyo University of Science, SUWA, Japan \\ jgh12701@ed.suwa.tus.ac.jp, shun@rs.suwa.tus.ac.jp
}

\begin{abstract}
Recently, Japan (also world-wide countries) has become aged society, and wide variety welfare device and system have been developed. But evaluation of welfare system and device are limited only stability, intensity and partial operability. So, evaluation of usefulness is insufficient. Therefore, we will attempt to establish the standard to evaluate usefulness for objectively and quantitatively on the basis of including non-verbal cognition. In this paper, we measure load of sitting and standing movement to use EMG (Electoromyogram) and 3D Motion Capture and set a goal to establish objective evaluation method. We think that establishing objective evaluation method is necessity to develop useful welfare device. We examined possibility of assessing load and fatigue from measuring brain activity to use NIRS (Near Infra-Red Spectoroscopy).
\end{abstract}

Keywords: Evaluation, Movement, Exercise, 3D Motion Capture, NIRS, EMG, Care, Welfare Technology, Evaluation method.

\section{Introduction}

As increasing aging population in Japan and world-wide countries, welfare systems and device are rapidly developing, and various devices are manufactured based on the increased popularity of welfare device and system. Also the market of welfare device and system are expanding. However, the evaluation method is limited respectively to stability, strength and a part of operability for individual system or device. It means that evaluation methodology for usefulness of them was not established. Therefore, we will attempt to establish the standard to evaluate usefulness for objectively and quantitatively on the basis of cognition such as physical load, reduction of fatigue and postural stability. Especially, in considering universality, it is necessary to measure human movement and brain activity in daily life. Also, we looked into the possibility of quantitative evaluation of tiredness and load on the basis of brain activity using NIRS. Also, we consider that physical and psychological load are linked to cognition including non-verbal cognition. In this paper, the purpose of experiments is to evaluate motion focusing on sitting and standing movement which is usually done in our life by using NIRS. We consider that human feel physical and psychological load during life motion. We tried to measure non-verbal cognition about psychological load by using NIRS. 


\section{Experimental Method}

We measured brain activity during motion with the purpose of establishing evaluation method based on generality (Fig. 1). Subjects were six males aged twenties. They were asked to read and sign an informed consent regarding the experiment too. Measurement apparatus was NIRS (SHIMADZU CO. Ltd products-FOIRE3000). Measurement region was at right and left prefrontal cortex.

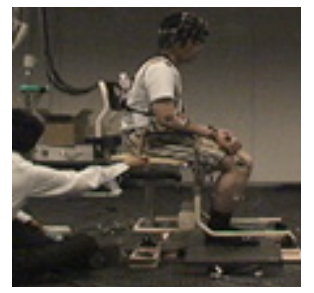

Fig. 1. Experimental view of NIRS

Measuring Brain Activity during Transfer with Standing Position (task1). At this measurement, subject used welfare device to perform transferring in a standing position. In this measurement, subject sat on seating face of welfare device appeared on the top of chair after raising hip until kneeling position. Also, subject performed inverse transferring from seating face to chair. Time design was rest (5 seconds) - task (10 seconds) - rest (5 seconds). This time design was repeated 30 times.

Measuring Brain Activity during Transfer with Half-Crouch Position (task2). At this measurement, subject used welfare device to perform transferring in a half-crouch position. In this measurement, subject sat on seating face of welfare device appeared on the top of chair after raising hip until kneeling position. Also, subject performed inverse transfer from seating face to chair. Time design was rest ( 5 seconds) - task (10 seconds) - rest (5 seconds). This time design was repeated 30 times. In experiments of task1 and task2, operation of welfare device was performed by operator other than subject. Before this measuring, subjects adjusted to transferring by use of welfare device.

Measuring Brain Activity during Keeping Half-Crouch Position (task3). Subjects performed two tasks at this measurement. During task3-1, they sat on seating face of welfare device with eyes open. During task3-2, they kept a half-crouch position. Subjects alternated task3-1 and task3-2. Also, subjects took resting time between two types of motion with eyes close. Therefore time design was rest ( 5 seconds) - task31 (10 seconds) - rest ( 5 seconds $)$ - task3-2(10 seconds) - rest (5 seconds). This time design was repeated 15 times. 


\section{Experimental Result}

As common result of all subjects, oxy-Hb tended to increase during task and to decrease in resting state. Therefore, it was thought that change of hemoglobin density due to task was measured. Fig. 2, 3 and 4 shows trend of the channel in which significant different was shown. Analysis was performed via one-sample t-test by a method similar to previous researches $[1,2,3,4,5]$. In this analysis, it was necessary to remove other than change of blood flow due to fatigue. So, our method was mainly focused on resting state to compare with the 1st trial and other trials of brain activity. In task1 1 and 2, each of sample data for analysis was 4 seconds after the task. In task 3 , sample data was 4 seconds during task. In the t-test of the same task, we performed t-test with first time trial and other trial which was from second times to thirty times, and examined relationship the number of trials and significant differences.

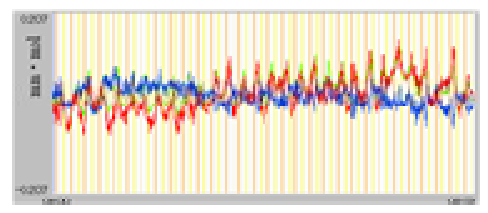

Fig. 2. Measuring Result of Task1

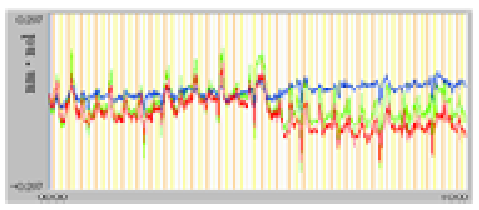

Fig. 3. Measuring Result of Task2

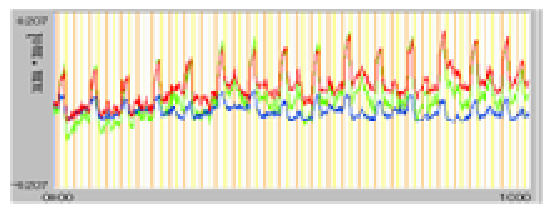

Fig. 4. Measuring Result of Task3
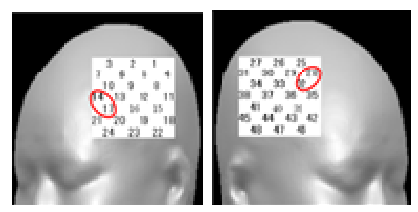

Fig. 5. Significant Difference of task1

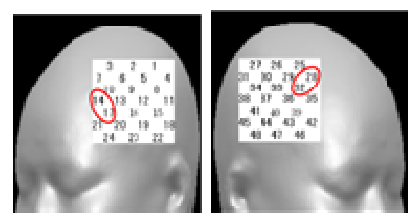

Fig. 7. Significant Difference of task 1 and 2

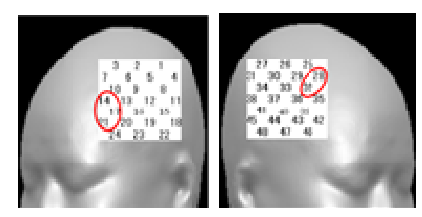

Fig. 6. Significant Difference of task2

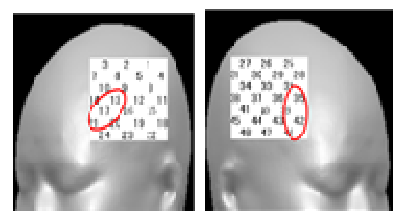

Fig. 8. Significant Difference in sitting position 
In task 1, significant different could be found from the about 10th trials. Fig. 4 show region confirmed significant different. In task 2 , significant different could be found from the about 10th trials too. Fig. 5 show region confirmed significant difference. Next, we performed t-test with case of standing position (task 1) and halfcrouch position (task 2). In this analysis, significant different could be found at prefrontal area $(14 \mathrm{ch}, 17 \mathrm{ch}, 28 \mathrm{ch}$ and $32 \mathrm{ch})$. Fig. 6 show region confirmed significant difference.

Also, two type of motion which was sitting and keeping a half-crouching position were repeated alternatively in task 3 . At first, we performed t-test using 4 seconds during first trial and 4 seconds during other trials which were from second to fifteenth in same position. Regarding to analysis result using sample data during sitting position and half-crouching position, there were significant different at Prefrontal area. Fig. 7 show region confirmed significant difference.

\section{$4 \quad$ Results and Verification Experiments}

In this experiment, we tried to measure quantitatively the physical and psychological strain on the basis of brain activity. Also, we think that brain activity disclose human cognitive including non-verbal. As a result, it was shown that there were differences at brain activity due to number of trials and postural. In this time, analysis was performed via one-sample t-test using sample of brain activity in resting state during task or after task. Hence, analysis method was to remove disturbance such as body motion and angular variation of neck to the extent possible although there was the possibility to measure skin blood flow. Therefore, it was thought that strain due to tasks was quantitatively measured by being recognized significant differences.

Also, in previous research, it was reported to decrease in activity in the brain around \#10, 11 [6] as the result of measuring brain activity during Advanced Trial Making Test using PET(Positron Emission Tomography) [7]. Therefore, this result came out in support of previous research in no small part.

Of course, it is necessary to increase number of subject at the present stage. In addition, there are problems associated with experiment, number of subject, method and measured region. However, in terms of being recognized significant differences at brain activity due to movement, it was thought to show useful result in evaluating quantitatively daily movements.

\section{Conclusion and Future Work}

We tried to measure physical and psychological load quantitatively on the basis of brain activity. And there were significant differences due to number of trials, holding position. In this experiment, analysis method was to remove disturbance such as body motion and angular variation of neck to the extent possible by using measurement result in resting state as sample. Therefore, it was thought to show useful result in evaluating quantitatively load due to movement task by being recognized difference in brain activity caused by number of trials, substance of task and holding position. 
Main purpose in this study is to evaluate physical load and fatigue quantitatively. So, we tried to evaluate change of muscle load due to difference of motion by simultaneous measuring with 3D motion analysis System and EMG quantitatively. However, evaluation of psychological load is necessary, too. In terms of using welfare device, prolonged use must be taken into account. In this case, it is important to consider not only physical load but also psychological load due to prolonged use from standpoint of developing welfare device and keeping up surviving bodily function. And, in previous research, separation between physical and psychological load has been performed. But, our view is that there is correlation with physical and psychological load. So, we tried to measure psychological load including physical one based on brain activity and quantitatively evaluate both load. For the future, our aim is to establish method of discussing useful of welfare device by evaluating load involved in other daily movements with increasing number of subjects.

\section{References}

1. Takahashi, N., Shimizu, S., Hirata, Y., Nara, H., Miwakeichi, F., Hirai, N., Kikuchi, S., Watanabe, E., Kato, S.: Fundamental Study for a New Assistive System during Car Driving. In: Proc. of International Conferrence on Robotics and Biomimetics, China (2010)

2. Takahashi, N., Shimizu, S., Hirata, Y., Nara, H., Inoue, H., Hirai, N., Kikuchi, S., Watanabe, E., Kato, S.: Basic study of analysis of human brain activities during car driving. In: Smith, M.J., Salvendy, G. (eds.) HCII 2011, Part I. LNCS, vol. 6771, pp. 627-635. Springer, Heidelberg (2011)

3. Shimizu, S., Takahashi, N., Nara, H., Inoue, H., Hirata, Y.: Fundamental study for human brain activity based on the spatial cognitive task. In: Hu, B., Liu, J., Chen, L., Zhong, N. (eds.) BI 2011. LNCS, vol. 6889, pp. 218-225. Springer, Heidelberg (2011)

4. Shimizu, S., Takahashi, N., Nara, H., Inoue, H., Hirata, Y.: Basic Study for Human Brain Activity Based on the Spatial Cognitive Task. In: The Third International Conference on Advanced Cognitive Techonologies and Applications, Italy (2011)

5. Shimizu, S., Takahashi, N., Inoue, H., Nara, H., Miwakeichi, F., Hirai, N., Kikuchi, S., Watanabe, E., Kato, S.: Basic Study for a New Assitive System Based on Brain Activity associated with Spatial Perception Task during Car driving. In: Proc. International Conferrence on Robotics and Biomimetics, Thailand (2011)

6. Watanabe, Y.: Molecular/neural mechanisms of fatigue and the way to overcome fatigue. Folia Pharmacological Japonica 129, 94-98 (2007)

7. Kuratsune, H., Yamaguti, K., Lindh, G., Evengard, B., Hagberg, G., Matsumura, K., Iwase, M., Onoe, H., Takahashi, M., Machii, T., Kanakura, Y., Kitani, T., Langstrom, B., Watanage, Y.: Brain Regions Involved in Fatigue Sensation: Reduced Acetylcarnitine Uptake in to the Brain. Neuroimage 17, 1256-1265 (2001)

8. Maruta, K.: The influence of Seat Angle on Forward Trunk Inclination During Sit-to-Stand. Jounal of Japanese Physical Therapy Association 31(1), 21-28 (2004) 